\title{
A 30 AÑOS DE LA CARRERA DE CIENCIAS DE LA EDUCACIÓN DE LA UNIVERSIDAD NACIONAL DE ROSARIO: "DIÁLOGOS, DEBATES Y PERSPECTIVAS EN EL CAMPO DE LA EDUCACIÓN"
}

\author{
Delfina Alodi* \\ Universidad Nacional de Rosario, Argentina. \\ delfi_1495@hotmail.com \\ María Isabel Pozzo* \\ Universidad Nacional de Rosario - CONICET, Argentina. \\ pozzo@irice-conicet.gov.ar
}

Recibido: 30/09/2017 Aceptado: 29/10/2016

\section{Resumen}

El presente escrito se propone dar testimonio de las Jornadas tituladas: "A 30 años de la Carrera de Ciencias de la Educación: Diálogos, debates y perspectivas en el campo de la educación" de la Escuela de Ciencias de la Educación de la Facultad de Humanidades y Artes perteneciente a la Universi-

* Estudiante de la Escuela de Ciencias de la Educación de la Universidad Nacional de Rosario

* Profesora Adjunta de Trabajo de Campo, Escuela de Ciencias de la Educación de la Universidad Nacional de Rosario. Docente-investigadora categoría II (Secretaría de Políticas Universitarias). Investigadora Categoría Independiente del Consejo Nacional de Investigaciones Científicas y Técnicas (CONICET) en el Instituto Rosario de Investigaciones en Ciencias de la Educación (IRICE). Profesora, Licenciada y Doctora en Ciencias de la Educación (Universidad Nacional de Rosario) y Magister por la Universidad de Barcelona. 
dad Nacional de Rosario. Dicho evento tuvo lugar el 12 y 13 de mayo de 2016 en el emblemático edificio de la Facultad antes nombrada.

Palabras Clave: Ciencias de la Educación - Universidad - Democracia - Universidad Nacional de Rosario.

\section{Abstract}

This article attempts to attest the Conference entitled: " $30^{\text {th }}$ anniversary of the Education Sciences Career: Dialogues, debates and perspectives in the field of Education" of the School of Educational Sciences, Faculty of Humanities and Arts that belongs to the National University of Rosario. The event took place in May 12th and 13th, 2016, in the emblematic building of the above mentioned Faculty.

Keywords: Educational Sciences - University - Democracy - National University of Rosario.

\section{A 30 AÑOS DE LA CARRERA DE CIENCIAS DE LA EDUCACIÓN DE LA UNIVERSIDAD NACIONAL DE ROSARIO: "DIÁLOGOS, DEBATES Y PERSPECTIVAS EN EL CAMPO DE LA EDUCACIÓN"}

Las fructíferas Jornadas del 12 y 13 de mayo del 2016 organizadas por la Escuela de Ciencias de la Educación, la Facultad de Humanidades y Artes y la Asociación de Graduados en Ciencias de la Educación de Rosario conformaron un escenario de encuentros, debates y discusiones propicias para el evento convocante. Durante las charlas-debates se repasaron los 30 años transcurridos desde la creación de la carrera, explicitándose el contexto de surgimiento de la misma. Incluso, se trataron sus proyecciones en el campo de la investigación, las luchas por la defensa de la educación pública, las diversas alternativas educativas y las problemáticas en torno a la educación del pasado, presente y futuro.

El momento en el que emerge esta carrera universitaria se caracterizó por el fervor del retorno de la democracia. Más allá de este sustrato común, las miradas de alumnos y profesores fueron multidiferenciales. Expresiones tales como educación interactiva, curriculum abierto, construcción del conocimiento, interdisciplinariedad, participación e investigación brotaron desde el subsuelo más profundo de esta Facultad y trajeron consigo un aire de esperanza después de un periodo tan cruel como el régimen de facto. La estruc- 
tura del plan de estudio -dividido en problemáticas, núcleos y áreas- está íntimamente ligada a estos conceptos que comenzaron a visibilizarse en dicha etapa. Paradójicamente a este intenso comienzo de la carrera, sus primeros graduados prorrumpieron en una época de gran decaimiento definida como "neoliberalismo", con fuerte impacto en la educación. Los conceptos que subyacían en aquel momento de origen poco tenían que ver con la nueva realidad que enfrentaba nuestro país. Los investigadores no eran valorados en su trabajo, los planes de estudio se organizaban fraccionadamente y los recursos escaseaban. El Estado se desentendía del sistema educativo, que quedaba a merced de provincias poco preparadas para sustentarlo.

Durante el panel de apertura de las Jornadas se dieron diversas discusiones, tales como la globalización y su resultado (la mercantilización del conocimiento), expuesto por el Lic. Gustavo Bruffman; y la disparidad que genera el capitalismo, definida por el Dr. Estanislao Antelo como la desigualdad que posee nuestra sociedad y el "amor" por la evaluación. Por su parte, la Mg. Norma Placci planteó su mirada acerca de los diversos roles del cientista de la educación. En los discursos se evidenciaron también las resistencias que generan algunas palabras, tales como inclusión, democracia escolar, aprendizajes alternativos, praxiología. Estas no son más que las pasiones del alma sumadas a la puja de poder y el conflicto de intereses.

Durante las Jornadas también tuvo su espacio la Revista de Ciencias de la Educación y el lanzamiento de su nuevo número. Se recordaron sus inicios en el año 2005, cuyo primer número, peculiarmente, fue el cero. Los distintos tomos contienen variedades de ensayos, investigaciones y reseñas, no sólo del ámbito nacional sino también del internacional. Desde sus inicios, la dirección está a cargo de la Mg. Susana Copertari, quien realiza un exhaustivo trabajo junto a su equipo para lograr que este proyecto perdure en la actualidad.

El programa contempló asimismo otras sesiones plenarias dedicadas a: la Biblioteca de la Escuela "Prof. Carlos Fuentealba", un sentido homenaje a los fundadores ya desaparecidos de la carrera (Prof. Leonor Bella de Paz y Dr. Ovide Menin), y un reconocimiento a las directoras de estas tres décadas. Asimismo, mesas paralelas focalizadas en temas precisos relativos al rol del cientista de la educación en las instituciones educativas, en espacios alternativos y en la investigación, coordinadas por profesores y graduados de la carrera.

Finalmente, es preciso nombrar la visita de la prestigiosa Dra. Adriana Puiggrós a nuestra casa de estudios. La disertante hizo referencia a temáticas como la inclusión desde el punto de vista de la transformación, la influencia de las corporaciones en el sistema educativo, la Ley Nacional de Educación Superior vigente -que desde hace años se está intentado reformular-, la meritocracia como herramienta escolar y la necesidad de verbalizar las diferencias y articularlas. 
A modo de cierre, se debe destacar la invalorable labor de los organizadores para que estas Jornadas, tan importantes para el desarrollo profesional de alumnos y docentes, puedan ser vistas como un punto de encuentro, de formulación de debates y discusiones, que como resultado generen amigos, según lo expresado por la Dra. Puiggrós. Cabe recuperar una frase que el Dr. Adrián Ascolani enunció como una crítica constructiva a la tarea de los cientistas de la educación: "Muchos estudian el pasado, varios piensan el futuro, pero pocos se focalizan en el presente". Estudiar el pasado es un quehacer arduo que nos ayuda a mirar el presente; pero mirar el futuro sin poder resolver problemáticas del presente es caer en oscuras tinieblas, que penosamente ya hemos atravesado en nuestro sistema educativo. 
